\title{
Correction to: Exploring Symptom Fluctuations and Triggers in Myalgic Encephalomyelitis/Chronic Fatigue Syndrome Using Novel Patient-Centred N-of-1 Observational Designs: A Protocol for a Feasibility and Acceptability Study
}

\author{
Suzanne McDonald ${ }^{1}$ (i) $\cdot$ Samuel X. $\operatorname{Tan}^{1}$ (i) $\cdot$ Shamima Banu ${ }^{1,2}$ (i) $\cdot$ Mieke van Driel ${ }^{2}$ (D) James M. McGree $^{3}$ (D) \\ Geoffrey Mitchell ${ }^{1,2}$ (D) Jane Nikles ${ }^{1}$ (D)
}

Published online: 25 September 2021

(c) The Author(s), under exclusive licence to Springer Nature Switzerland AG 2021

\section{Correction to: \\ The Patient - Patient-Centered Outcomes Research https://doi.org/10.1007/s40271-021-00540-0}

In this article the reference to the second to last sentence of the first paragraph of section 2.6.2 was incorrect.

It should have been,
Hickey A, Newham J, Slawinska MM, Kwasnicka D, McDonald S, Del Din S et al. Estimating cut points: a simple method for new wearables. Maturitas. 2016;83:78-82.

The original article has been corrected.

The original article can be found online at https://doi.org/10.1007/ s40271-021-00540-0.

Suzanne McDonald suzanne.mcdonald@uq.edu.au

1 Centre for Clinical Research, The University of Queensland, Brisbane, QLD, Australia

2 Faculty of Medicine, The University of Queensland, Brisbane, QLD, Australia

3 Science Faculty, Queensland University of Technology, Brisbane, QLD, Australia 